technology and RNA interference (RNAi).

In the realm of drug discovery, antisense technology - the use of short oligonucleotides to target specific messenger RNAs for destruction was developed as a way of finding oligonucleotide-based drugs that interfere with gene expression, rather than with protein function. But the technology is currently enjoying greater success as a highthroughput method of target validation because it offers a highly specific and efficient way to inhibit the expression of potential target proteins in vitro and in vivo.

GeneTrove, the genomics division of Isis Pharmaceuticals in Carlsbad, California, is one of the companies active in this field. It is focusing on the untapped pool of potential therapeutic target RNAs for both target validation and drug discovery, says Nicholas Dean, GeneTrove's vice-president of functional genomics. It offers custom targetvalidation packages that include optimized antisense inhibitors against any target of interest and control oligonucleotides for testing in cell-culture model systems. It also applies antisense technology to target validation in vivo in animal models.

Biognostik, a biotechnology company in Göttingen, Germany, offers a drugtarget validation kit that can be used in vitro or in vivo. It includes five targetspecific phosphorothioate antisense inhibitors and two random-sequence oligonucleotides to control for nonspecific

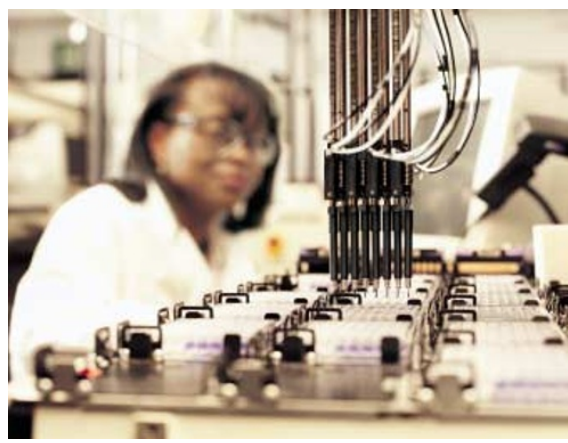

GeneTrove analyses the effects of antisense on gene expression.

effects. It has also developed a sequencedesign system called RADAR, which determines antisense oligonucleotides based on specificity, minimal nonspecific effects or protein binding, and the ability to be taken up into cells.

Sequitur, a functional-genomics company in Natick, Massachusetts, has a slightly different approach to rapid target validation. It combines an antisense library with high-throughput DNA microarray assays to test the effects of the antisense molecules on gene expression. The company's technology was used recently to validate a major therapeutic target for Alzheimer's disease. Sequitur also carries out target validation based on RNAi (see 'The silent treatment', page 343).

Custom phosphorothioate antisense oligonucleotides for research are available from firms such as Sigma-Genosys at the Woodlands, Texas; atugen in Berlin, Germany; and Integrated DNA Technologies in Coralville, Iowa. Gene Tools in Philomath, Oregon, offers morpholino antisense oligonucleotides, and Danish companies Cureon in Copenhagen and Exiqon in Vedbæk offer modified oligonucleotides based on 'locked nucleic acid' technology that can be used for antisense.

\section{The proteomics approach}

One disadvantage of doing target validation at the genetic level is that many genes produce several different protein isoforms, which can have subtly different functions (see 'A question of form', page 341). Posttranslational modifications can also give protein variations. As a result, a developing approach in target validation is to focus on manipulating the activity of the potential target protein itself. "As the vast majority of drugs target proteins, validating targets is best done by modulating protein activity, not expression levels," Szymkowski says.

Proteomics - the study and manipulation of the protein make-up of a cell — is making it easier to distinguish and target just one specific form of a protein. This allows researchers to avoid unwanted changes in the expression of other proteins - another potential drawback of genetic manipulations.

Stefan Henning, director of functional biology at Xerion Pharmaceuticals in

\title{
A WHOLE PICTURE
}

Beyond Genomics in Waltham, Massachusetts, is taking a broad view for target validation - its approach hinges on systems biology. The company first characterizes potential targets within their normal biochemical pathways. "We measure cells, tissues and body fluids at multiple bioanalytical levels, including transcript, protein, and endogenous metabolite and enzyme product," says Aram Adourian, the company's senior director of advanced technologies and strategic development.

It then compares these profiles with similar profiles from disease states, and correlates features of the profile with the disease in question using pattern-recognition algorithms and bioinformatics techniques. The result is a set of potential targets, which can then be perturbed by RNAi or transgenics, for example. The subsequent biological changes are monitored by the same systems-biology approach.

As a target-validation tool, systems biology is "a truly exciting method for illuminating causative relations between target modulation and effects on disease", says Adourian. By learning more about the biological pathways in which the target is involved, it is possible to predict the potential toxicity of perturbing its normal function, he says.

Adourian believes that an integrative approach to target validation is more likely to yield the highest-quality targets: "One ultimately needs to examine the system as a whole, with its interacting networks and components of genes, proteins, metabolites and other molecules, to begin to assemble a unified and contextualized perspective on the role, function and relevance of a potential target."
Another firm taking a wide view of target validation is MDS Proteomics in Toronto, Canada, which uses bioinformatics, gene-expression information, high-throughput protein analysis and protein-pathway biology to identify prospective targets and gain all-round evidence of their cellular role.

c.s.

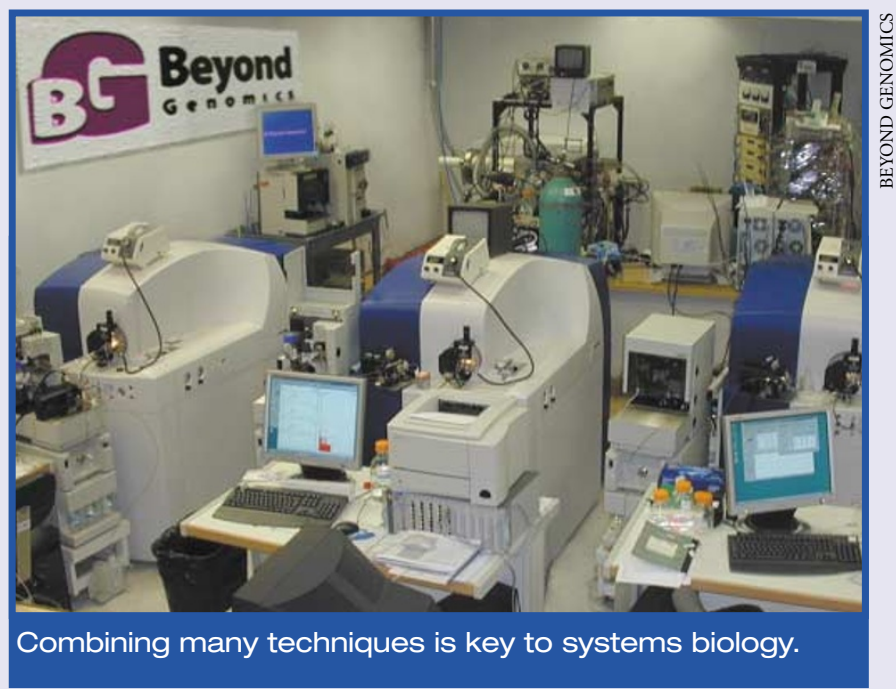

